Access to this work was provided by the University of Maryland, Baltimore County (UMBC)

ScholarWorks@UMBC digital repository on the Maryland Shared Open Access (MD-SOAR) platform.

Please provide feedback

Please support the ScholarWorks@UMBC repository by emailing scholarworks-group@umbc.edu and telling us what having access to this work means to you and why it's important to you. Thank you. 
Research article

\title{
A novel model for the RNase MRP-induced switch between the different forms of 5.8S rRNA
}

\author{
Xiao $\mathrm{Li}^{1,2,3}$, Janice M Zengel ${ }^{1}$ and Lasse Lindahl ${ }^{1}$ \\ ${ }^{1}$ Department of Biological Sciences, University of Maryland Baltimore County (UMBC) \\ 1000 Hilltop Circle, Baltimore, Maryland 21250, USA \\ 2 Department of Biology, University of Rochester, Rochester, New York 14627, USA \\ ${ }^{3}$ Current address: Abbott Laboratories, San Diego, CA
}

ribosome biogenesis; rRNA processing; RNase MRP; long/short 5.8S rRNA 
Abstract: Processing of the RNA polymerase I pre-rRNA transcript into the mature 18S, $5.8 \mathrm{~S}$, and $25 \mathrm{~S}$ rRNAs requires removing the "spacer" sequences. The canonical pathway for the removal of the ITS1 spacer, located between 18S and 5.8S rRNAs in the primary transcript, involves cleavages at the 3 ' end of 18S rRNA and at two sites inside ITS1. The process generates a long and a short 5.8S rRNA that differ in the number of ITS1 nucleotides retained at the 5.8S 5 ' end. Here we document a novel pathway that generates the long $5.8 \mathrm{~S}$ for ITS1 while bypassing cleavage within ITS1. It entails a single endonuclease cut at the 3'-end of 18S rRNA followed by exonuclease Xrn1 degradation of ITS1. Mutations in RNase MRP increase the accumulation of long relative to short 5.8S rRNA; traditionally this is attributed to a decreased rate of RNase MRP cleavage at its target in ITS1, called A3. In contrast, we report here that the MRP induced switch between long and short 5.8S rRNA formation occurs even when the A3 site is deleted. Based on this and our published data, we propose that the switch may depend on RNase MRP processing RNA molecules other than pre-rRNA. 


\section{Introduction}

Ribosome formation is the most resource-requiring process in both pro- and eukaryotes [1-3]. It involves complex pathways for regulated synthesis of rRNA and ribosomal proteins (r-proteins), and the assembly of these components into functional ribosomal subunits. The pathways differ between pro- and eukaryotes, but in eukaryotes the major steps are conserved from yeast to humans, although the complexity has evolved [4-6].

The progression of eukaryotic ribosome formation has been most intensively studied in Saccharomyces cerevisiae ('yeast') [7-9]. In this work, we focus on the processing of the yeast rRNA. Three of the four eukaryotic rRNA molecules are generated from a single precursor rRNA (pre-rRNA), polymerized by RNA Polymerase I (Pol I), that contains the sequences for $18 \mathrm{~S}, 5.8 \mathrm{~S}$ and $25 \mathrm{~S}$ rRNA separated by the internal transcribed spacers ITS1 and ITS2, and flanked by 5' and 3' external transcribed spacers (ETS) (Figure 1A). The pre-rRNA is processed by endo- and exonucleases to form the mature rRNAs (Figure 1B) concurrently with the assembly of the rRNA and 79 r-proteins into the two ribosomal subunits [9]. The rRNA processing can take place during transcription or after completed synthesis of the pre-rRNA transcript $[10,11]$. 


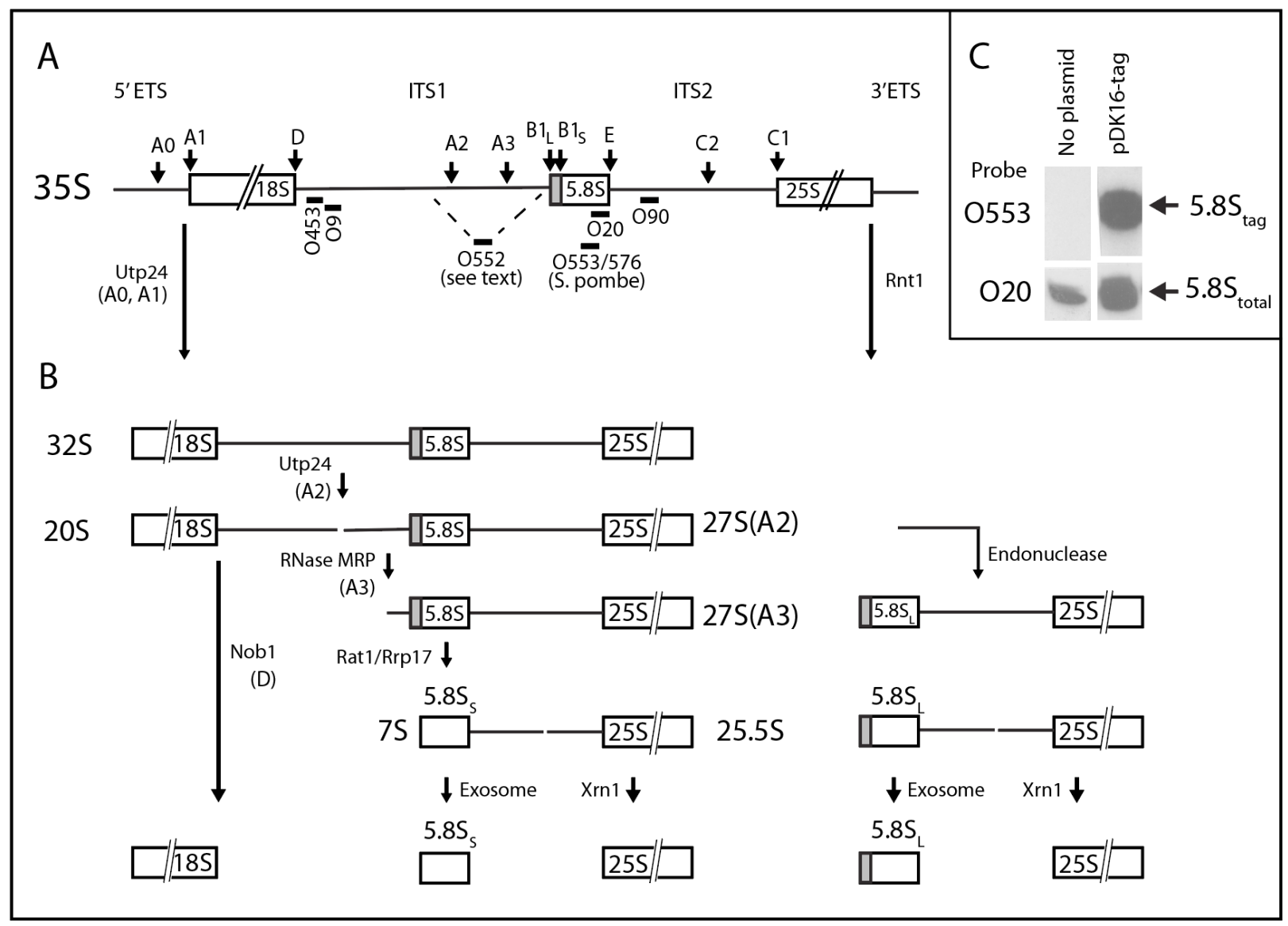

Figure 1. Canonical rRNA processing pathways. (A) Map of the yeast rRNA transcription unit with processing sites (above map) and oligonucleotides used for probing northern blots (below map). See Table 1 for oligonucleotide sequences and positions of pre-rRNA to which they hybridize. (B) rRNA processing intermediates with names of relevant processing enzymes and their sites of action. Only relevant steps are shown. (C) Specificity of northern probe for tagged 5.8S rRNA. $\Delta r n a 12$ without plasmid or carrying pDK16-tag wildtype was grown at $25^{\circ}$ and shifted to $37^{\circ}$ for 6 hours. Total RNA was analyzed by northern analysis using the 0553 probe (complementary to the $S$. pombe tag in 5.8S rRNA on pDK16-tag) or the 020 probe (complementary to the 25 nucleotides at the 3 ' end of $5.8 \mathrm{~S}$ rRNA).

The canonical model for processing of the primary Pol I transcript begins with the Utp24 and Rnt1 endonucleases splitting the 5' ETS and 3' ETS, respectively, from the main portion of the pre-rRNA, generating the 32S intermediate (Figure 1B) [12-14]. Utp24 then cuts at the A2 site in ITS1, which separates the rRNA moieties destined for the $40 S$ or the $60 S$ ribosomal subunit $[12,14,15]$. ITS1 is further processed to generate the 5 ' end of the 5.8S rRNA and the 3' end of 18S rRNA. 
There are two pathways that generate the 5' end of the 5.8S. In the major pathway, the ribozyme RNase MRP cuts ITS1 at the A3 site, followed by trimming of the resulting 5' end by a combination of Rat1 and Rrp17 exonucleases to form the 5' end of the "short" 5.8S rRNA (5.8Ss) (Figures 1 and 2) [16-21]. In the other pathway, an unknown endonuclease directly generates the 5 ' end of the "long" $5.8 \mathrm{~S}$ rRNA ( $5.8 \mathrm{~S} \mathrm{~L}$ ), which is 7 nucleotides longer at the 5 ' end than $5.8 \mathrm{Ss}$ [22]. Here we have identified an additional pathway to $5.8 S_{L}$ involving degradation of the entire ITS1 from its 5 ' end at cleavage site $D$ to the 5 ' end of $5.8 \mathrm{~S}$.

Deletion or mutational inactivation of the gene encoding the RNA subunit of both yeast and human RNase MRP results in incomplete rRNA processing, and blocks ribosome formation [23, 24]. However, it is not clear why RNase MRP is essential for ribosome production. Mutations impairing (but not eliminating) RNase MRP activity favor the accumulation of $5.8 \mathrm{~S}$, increasing the ratio between long and short 5.8S rRNA ( $L / S$ ratio) [20, 24-27]. It is generally believed that this is caused by a decreased rate of RNase MRP cleavage at $A 3$, which in turn reduces the number of $A 35^{\prime}$ ends available for attack by Rat1 and Rrp17. However, this would not explain why RNase MRP is essential for rRNA synthesis and growth, since both types of 5.8S rRNA can be incorporated into functional ribosomes.

Here we report a surprising result: namely, an RNase MRP affects the L/S ratio, even when the $A 3$ site is deleted from the pre-rRNA genes. This leads us to suggest that the role of RNase MRP in modulating the level of $5.8 \mathrm{~S}_{\mathrm{L}}$ is indirect and works through cleavage of non-rRNA targets, such one or more mRNAs encoding ribosome processing factors. The existence of such target(s) for RNase MRP may also help to explain why the enzyme is essential for survival of eukaryotes.

\section{Results}

\subsection{System for genetic analysis}

The S. cerevisiae genome has 100-200 tandem copies of the 18S-5.8S-25S rRNA transcription unit that is transcribed by RNA Polymerase I (Pol I). The large number of rRNA genes makes traditional genetic manipulation difficult and we therefore used strains in which Pol I is inactivated either permanently ( $\Delta$ rna135) or conditionally at 
$37^{\circ}$ (Arna12) [28, 29] (Table 2). Both strains harbor a 18S-5.8S-25S rRNA transcription unit on the high-copy plasmid pDK16 that is transcribed by RNA polymerase II (Pol II) from the $\mathrm{Cu}^{2+}$ induced CUP1 promoter [30]. Previous studies showed that pre-rRNA transcripts produced by Pol II are processed through the same steps as the natural Pol I transcripts $[28,30]$.

To specifically identify 5.8S rRNA transcribed from the plasmid-borne Pol IIdriven rRNA genes, we tagged the 5.8S rRNA derived from pDK16 by replacing the DNA sequence corresponding to a hairpin formed by nucleotides 124-147 of the $5.8 \mathrm{~S}$ rRNA with the DNA sequence that generates the same hairpin in 5.8S rRNA Schizosaccharomyces pombe (Sp), albeit with a different nucleotide sequence. The modified 5.8S rRNA transcribed from this plasmid, called pDK16-tag, was detected on northern blots by probing with an oligonucleotide (O553 or O576) that is complementary to the $S$. pombe sequence (Table 1). Total 5.8S rRNA transcribed from both wildtype and tagged genes was visualized on northern blots by a probe, $\mathrm{O} 20$, that is complementary to the 25 nucleotides at the 3 ' end of $5.8 \mathrm{~S}$ rRNA and thus present in both wildtype and Sp-tagged 5.8S rRNA (Figure 1C).

Table 1. Sequence of oligonucleotides used

\begin{tabular}{|l|l|l|}
\hline Oligo & Sequence & Complementary to \\
\hline O9 & GCT CTT GCC AAA ACA AAA AAA TCC A & ITS1 24-53 \\
\hline O20 & AAA TGA CGC TCA AAC AGG CAT GCC C & $5.8 S$ ' end \\
\hline O90 & GTA TCA CTC ACT ACC AAA CAG AAT G & ITS2 11-35 \\
\hline O453 & AAC AAA AAA ATC CAT TTT CAA & ITS1 15-25 \\
\hline O552 & CCA GTT ACG CGT TTT AAT TG & Spanning $\Delta 2$ deletion, see text \\
\hline O553 & ATG CCT TTG GTA GAA CCC AAA GGC & S. pombe hairpin inserted in S. cerevisiae 5.8S \\
\hline O576 & ATG CCT TTG GTA GAA CCC & S. pombe hairpin inserted in S. cerevisiae 5.8S \\
\hline
\end{tabular}

\subsection{Effects of ITS1 deletions on $5.8 S$ rRNA processing}

Previous experiments have shown that the canonical $A 2$ and $A 3$ cleavage sites in ITS1 are dispensable for formation of mature rRNA [18, 30]. To determine if other regions in ITS1 are necessary for 5.8S rRNA formation, we generated several ITS1 deletions in the rRNA transcript on pDK16-tag (Figure 2A). The deletion plasmids were transformed into the temperature-sensitive strain $\triangle r n a 12$ to test if the ITS1-rRNA deletion genes can support growth at the non-permissive temperature for RNA pol I. 
Somewhat surprisingly, only one deletion $(\Delta 4)$, removing 82 nucleotides in the upstream part of ITS1, was lethal at $37^{\circ}$, while a shorter deletion within the same region of ITS1 $(\Delta 7)$ resulted in severely impeded growth. All the remaining deletion plasmids supported growth at $37^{\circ}$ in the presence, but not in the absence, of $\mathrm{Cu}^{2+}$, the inducer of the CUP1 promoter driving transcription of the plasmid-borne rRNA transcription unit. 


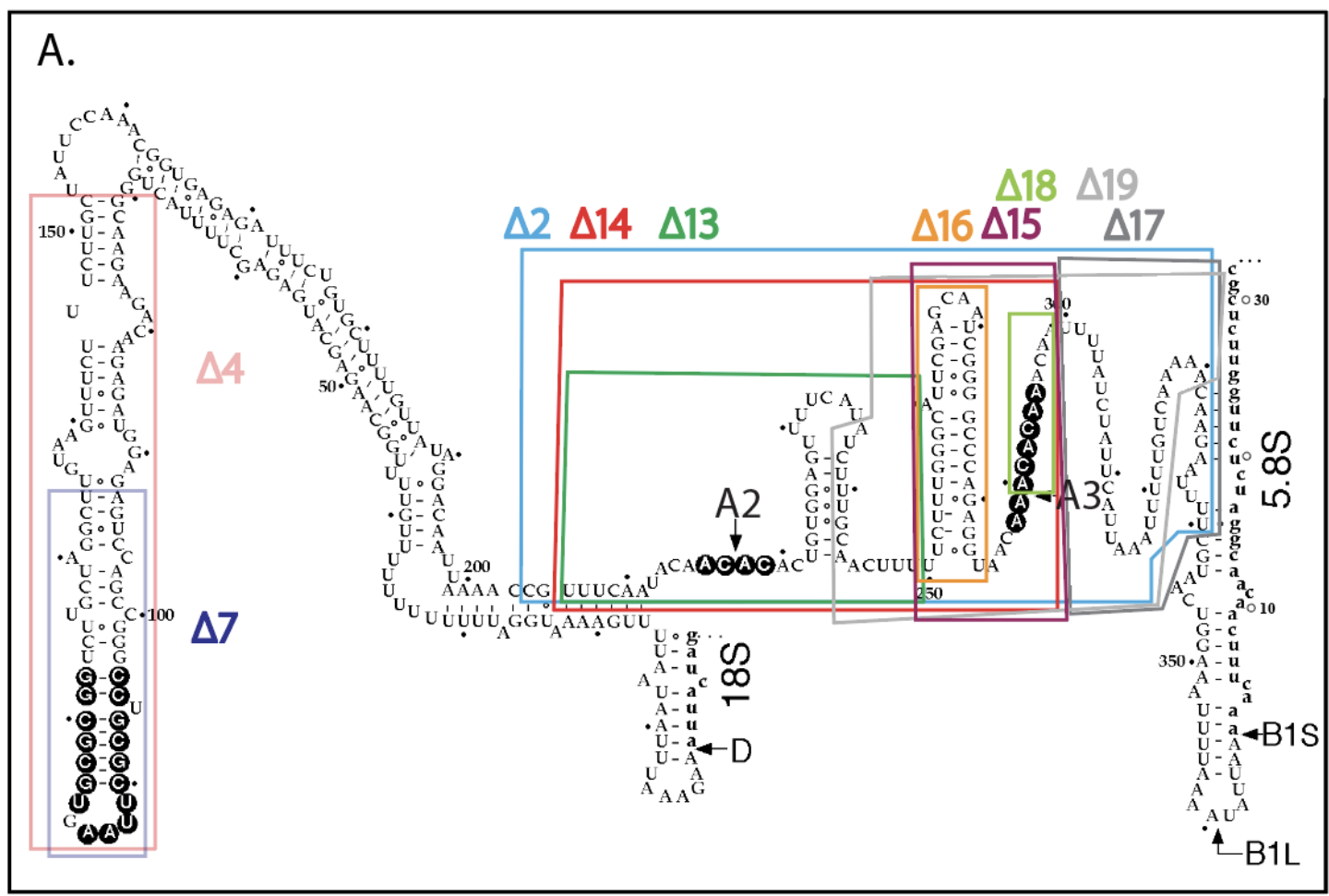

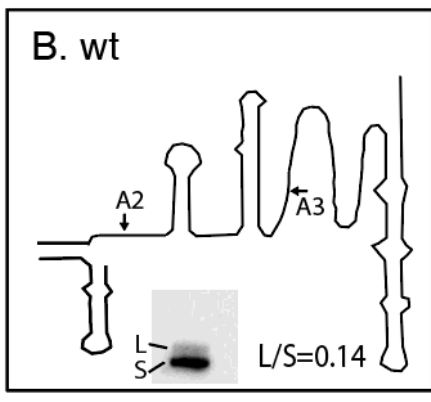
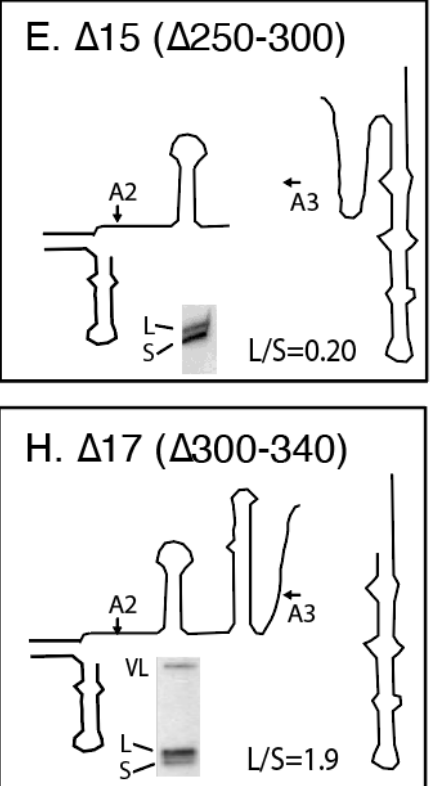
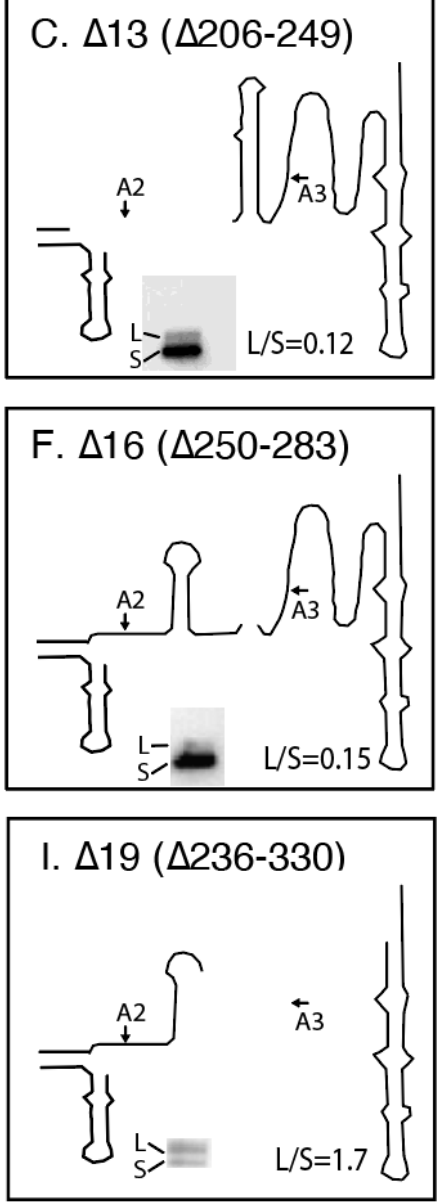

D. $\Delta 14$ ( $\Delta 206-299)$

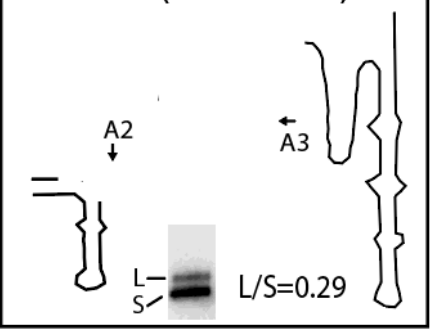

G. $\Delta 18(\Delta 290-299)$
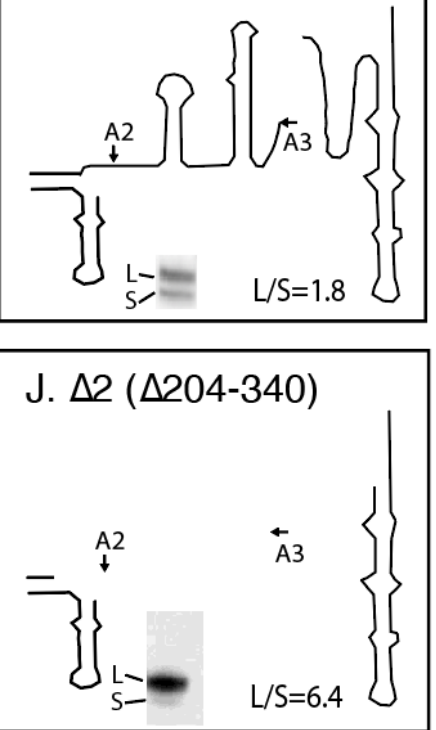
Figure 2. Deletion mutations in ITS1. (A) Secondary structure of ITS1 in S. cerevisiae proposed by van Nues at al. [31]. Deletions are shown by color-coded frames around the deleted nucleotides. Phylogenetically conserved nucleotides among a set of Saccharomycetales are shown in reverse contrast; see for specifics [31]. (B-J) Schematics of the ITS1 secondary structure in which the deleted parts in each mutant are blocked out. The ratio of $5.8 \mathrm{~S} \mathrm{~L}$ and $5.8 \mathrm{~S} \mathrm{~S} \mathrm{rRNA}$ (L/S ratio) was determined by growing cells at $25^{\circ}$, shifting to $37^{\circ}$ for 6 hours before analyzing total RNA on northern blots using 0553 as probe. The $5.8 \mathrm{~S}$ rRNA section of northern blots for each mutant is shown at the bottom of each frame together with the L/S ratio determined from the autoradiogram.

We next determined the effects of the viable deletions on the relative accumulation of the long $(5.8 \mathrm{~S} \mathrm{~L})$ and short $(5.8 \mathrm{~S} \mathrm{~s})$ rRNAs. Cultures of $\Delta r n a 12$ harboring pDK16-tag, or one of its ITS1 deletion derivatives, were grown at $25^{\circ}$ and then shifted to $37^{\circ}$ for 6 hours. Total RNA was extracted and analyzed by acrylamide gel electrophoresis and northern blot hybridization, using the oligonucleotide probe 0553 (complementary to the $5.8 \mathrm{~S} \mathrm{Sp} \mathrm{tag)} \mathrm{(Figure} \mathrm{2).} \mathrm{We} \mathrm{then} \mathrm{calculated} \mathrm{the} \mathrm{ratio} \mathrm{between}$ the long (5.8S L) and short (5.8Ss) 5.8S rRNA (L/S ratio) produced from the deletion prerRNAs. For wildtype ITS1, the ratio was 0.14 , i.e., the cells accumulated about 7 -fold more $5.8 \mathrm{~S}$ s than $5.8 \mathrm{~S} \mathrm{~L}$, consistent with the $\mathrm{L} / \mathrm{S}$ ratio in $5.8 \mathrm{~S}$ rRNA transcribed from chromosomal genes (Figure $2 B$ ) [27, 32]. Deletions removing the A2 site $(\Delta 13)$, the $A 2$ and $A 3$ sites together $(\Delta 14)$, the $A 3$ site by itself $(\Delta 15)$, or a proposed hairpin upstream of the $A 3$ site $(\Delta 16)$ all have no or modest (2-fold or less) effects on the L/S ratio (Figure $2 \mathrm{C}-\mathrm{F})$. However, an 11-nucleotide deletion immediately downstream of the A3 cleavage site $(\Delta 18)$ increased the L/S ratio by about 10 -fold (Figure $2 \mathrm{G}$ ). Importantly, a deletion that leaves the $A 3$ site intact, but removes 41 nucleotides downstream of the $A 3$ region $(\Delta 17)$ has a similar effect on the L/S ratio (Figure $2 \mathrm{H}$ ), suggesting that ITS1 sequences or structures downstream of $A 3$ are important determinants for the L/S ratio. The $\Delta 19$ deletion, which removes 55 nucleotides upstream through 40 nucleotides downstream of $\mathrm{A} 3$, also increases the L/S ratio by about the same factor (Figure 2I), but since $\Delta 14$ and $\Delta 15$ remove the $A 3$ site yet have little effect on the L/S ratio, it seems likely that the effect of the $\Delta 19$ deletion is due to removing ITS1 sequences or structures downstream of $A 3$ rather than removing the $A 3$ cleavage site itself. Note that $\Delta 14$ and $\Delta 15$ have no 
strong effect on the L/S ratio, even though they extend through the sequence removed by $\Delta 18$, which strongly increases the L/S ratio. This may suggest that $\Delta 18$ affects the L/S ratio by changing the structure of ITS1 downstream of $A 3$ rather that by removing the 11 nucleotides. Overall, the analysis the L/S ratios resulting from the ITS1 deletion mutant pre-rRNAs suggests that only sequences downstream of $A 3$, not sequences upstream of $\mathrm{A} 3$ or the presumed RNase MRP cleavage site itself, are necessary for maintaining the normal $\mathrm{L} / \mathrm{S}$ ratio.

While deletions removing ITS1 sequences downstream of the A3 cleavage site result in a 10-fold increase in the L/S ratio, the most striking increase was observed with mutant $\Delta 2$, in which the deletion of sequences downstream of $A 3$ is expanded to include the upstream A2 site (Figure $2 \mathrm{~J}$ ). To further analyze the $5.8 \mathrm{~S}$ accumulated in the $\Delta 2$ mutant, we used primer extension to map the 5 ' end of the plasmid-derived $5.8 \mathrm{~S} \mathrm{~L}$ from total RNA extracted from $\Delta r n a 135$ carrying pDK16 or pDK16 $\Delta 2$ (Figure 3). The results showed that no detectable $5.8 \mathrm{~S} s$ is generated from the $\Delta 2$ pre-rRNA. Furthermore, the 5 ' end of the $5.8 S_{L}$ is 7 nucleotides proximal to the $5^{\prime}$ end of the $5.8 \mathrm{~S}$ rRNA made from the rRNA genes on pDK16 without ITS1 deletions. This position matches the 5' end of $5.8 \mathrm{~S}\llcorner$ accumulating in RNase MRP mutants with wildtype chromosomal rRNA genes [27]. 


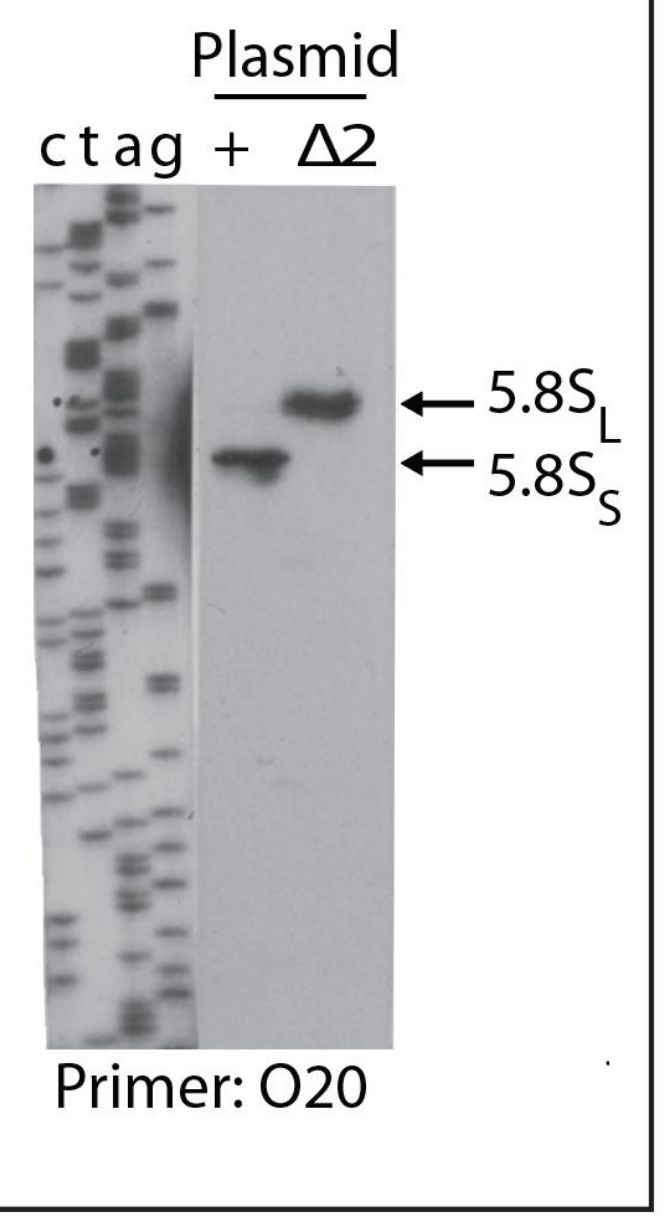

Figure 3. Mapping of 5' ends of 5.8S rRNA processed from $\triangle 2$ and wildtype rRNA genes carried on plasmid pDK16. The host strain $\Delta r n a 135$ carrying wildtype pDK16 or pDK16 $\Delta 2$ was grown in steady state at $30^{\circ}$. Total RNA was isolated and used for extension primer O20. Dideoxy sequencing ladders generated by extension of the 020 primer on the pDK16 DNA are shown as markers. Dots in the sequence lanes indicate the band in the sequence ladder lining up with the primer extension products.

2.3. pre-rRNA processing is altered by an RNase MRP mutation even when the ITS1 A3 site is deleted.

The 5.8S L/S ratio increases in RNase MRP mutants [18, 20, 25, 32]. This increase has been attributed to a reduced rate of A3 cleavage with an ensuing decrease in the number of A3 5' ends available for attack by Rat1/Rrp17 to generate 5.8Ss. If this explanation is true, then 5.8S rRNA formation in mutants lacking the A3 site should not be affected by RNase MRP mutations. To examine this, we transformed plasmid 
pDK16-tag and its deletion derivatives into isogenic strains (YLL53 and 54) containing either the wildtype gene for the RNase MRP RNA subunit (RRP2, alias NME1) or a temperature sensitive single base substitution mutant of the RNase MRP RNA, called rrp2-2 [20]. Total RNA from these strains was analyzed by northern blots probed with O553, which is specific to the 5.8S rRNA with the Sp tag. As expected, the L/S ratio of 5.8S rRNA synthesized from the pDK16-tag plasmid without an ITS1 deletion is increased in the rrp2-2 mutant relative to the RRP2 parent strain (Figure 4, compare lanes 1-2 and 13-14 with lanes 21-22 and 33-34, respectively). The L/S ratio is also increased in rrp2-2 harboring pDK16-tag $\Delta 13$ and $\Delta 16$ (Figure 4, compare lanes 5-6 and 15-16 with lanes 25-26 and 35-36, respectively); both of these deletions leave the A3 site intact. Remarkably, the rrp2-2 mutation also changes the relative amount of $5.8 \mathrm{~S} \mathrm{~L}$ in mutants $\Delta 14$ and $\Delta 15$ (Figure 4, compare lanes 7-8 and 9-10 with lanes 27-28 and 2930 , respectively), even though $A 3$ is deleted in these mutants. In contrast, the RNase MRP mutation has little effect on the L/S ratio in mutants $\Delta 2, \Delta 17$ and $\Delta 18$, which already make little $5.8 \mathrm{~S} s$ in the $R R P 2$ wildtype background (Figure 4, compare lanes 34, 17-18, and 19-20 with lanes 23-24, 37-8, and 39-40, respectively). These results corroborate the notion that ITS1 sequences downstream of A3 contain the most important determinants for the relative accumulation of long and short 5.8S rRNA. 


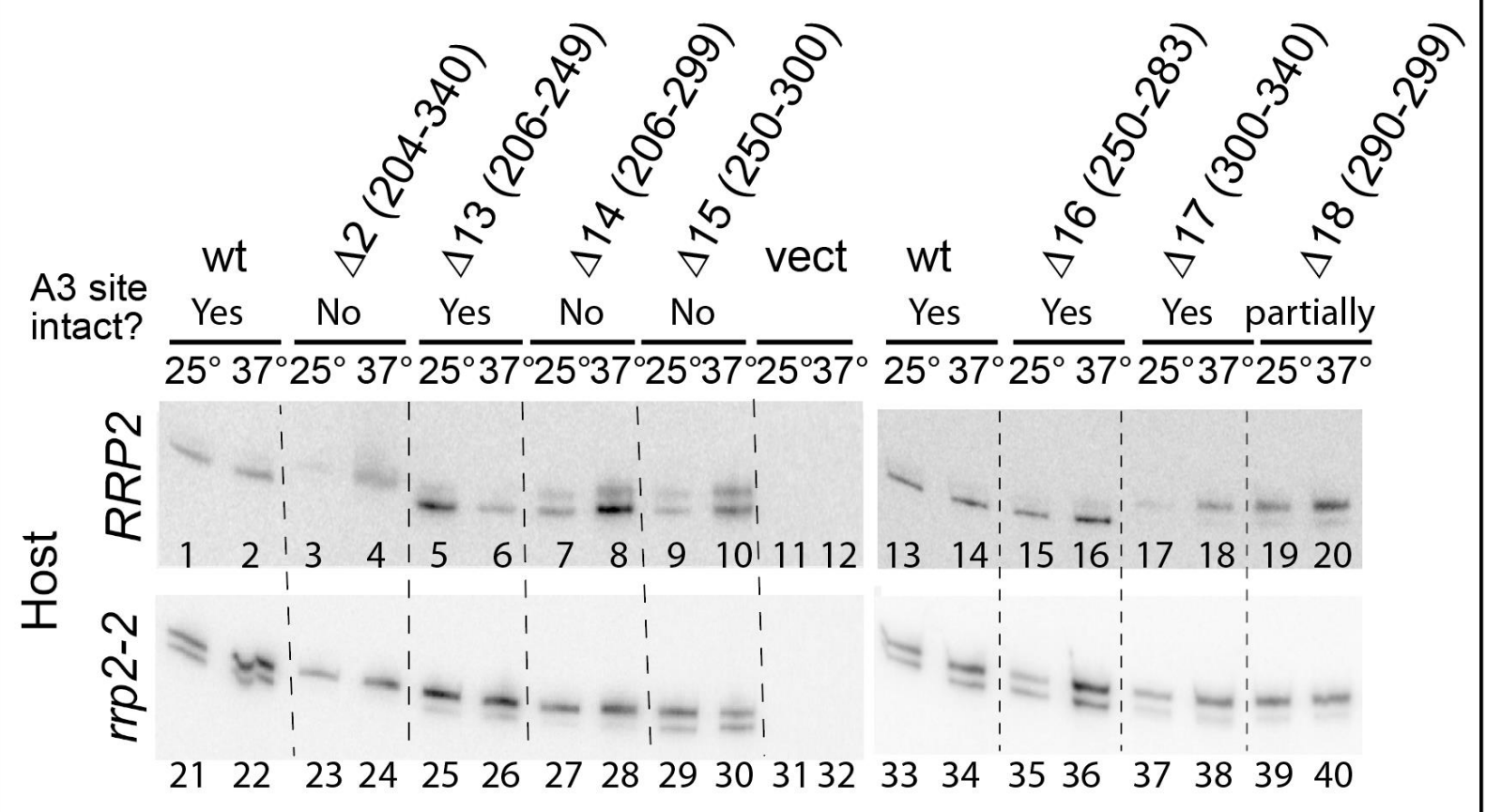

Probe:

O553

Figure 4. The effect of a mutation in the RNA subunit of the RNase MRP on the relative accumulation of $5.8 \mathrm{~S}_{\mathrm{L}}$ and $5.8 \mathrm{~S}_{\mathrm{S}} \mathrm{rRNA}$ during processing of pre-rRNA for wildtype and ITS1 mutants. Plasmid pDK16-tag carrying the indicated ITS1 deletion was transformed into isogenic strains with genes encoding the wildtype (RRP2) or mutant (rrp2-2) RNA subunit of the RNase MRP. Total RNA was prepared from each strain growing at $25^{\circ}$ and 6 hours after a shift to $37^{\circ}$ and analyzed by northern blots probed with 0553, which is specific for the 5.8S rRNA tag. The top band in each lane is the $5.8 \mathrm{~S}_{\mathrm{L}}$ and the lower band is $5,8 \mathrm{~S}_{\mathrm{S}}$. Note that the rrp2-2 strain grows at $25^{\circ}$, not at $37^{\circ}$, yet displays a similarly mutant RNA phenotype at both temperatures [27].

2.4. A pathway to 5.8S rRNA that bypasses the canonical A2 and A3 cleavage sites in ITS1

The accumulation of $5.8 S$ rRNA resulting from processing of the $\Delta 2$ and $\Delta 14$ mutants (Figures 2-4) shows that the canonical path to 5.8S rRNA via cleavage at A2 and/or A3 can be bypassed. To learn more about the bypass pathway(s), we first asked if the mutant pre-rRNAs are cleaved accurately at the D site at the 3 ' end of mature $18 \mathrm{~S}$ rRNA. Total RNA from $\Delta r n a 12$ cells harboring the pDK16-tag plasmid or the $\Delta 2, \Delta 13$, 
$\Delta 14$, and $\Delta 15$ deletion derivatives was prepared 6 hours after a shift from $25^{\circ}$ to $37^{\circ}$, at which time wildtype processing intermediates derived from residual transcription of the chromosomal rRNA genes are no longer visible (see below). Total RNA was used as template for extension of oligonucleotide 09, which hybridizes to ITS1 24-53 nucleotides downstream of the $D$ site (Figure $1 A$ and Table 1). RNA from the wildtype and the $\Delta 2, \Delta 13$, and $\Delta 14$ strains generated bands matching the 5 ' end of ITS1, adjacent to the 3' end of 18S rRNA (Figure 5, lanes 2-5). This result demonstrates that accurate cleavage at the $D$ site is not dependent on prior cleavage at A2 or A3.

Interestingly, in mutant $\Delta 15$ the band corresponding to cleavage at $D$ is absent; instead, there is a band corresponding to ectopic cleavage about 10 nucleotides downstream of the proper D site (Figure 5, lane 4, "D- 10"). (A lesser amount of the "D- 10" band is generated in $\Delta 13$ (Figure 5, lane 6), as well as the canonical $D$ band.) Since the deletion in $\Delta 15$ is more than 200 nucleotides downstream of $D$, it is not clear why this deletion abrogates proper $\mathrm{D}$ cleavage. Perhaps the loss or gain of a particular secondary or tertiary structure in $\Delta 15$ ITS1 affects the accessibility of the cleavage site.

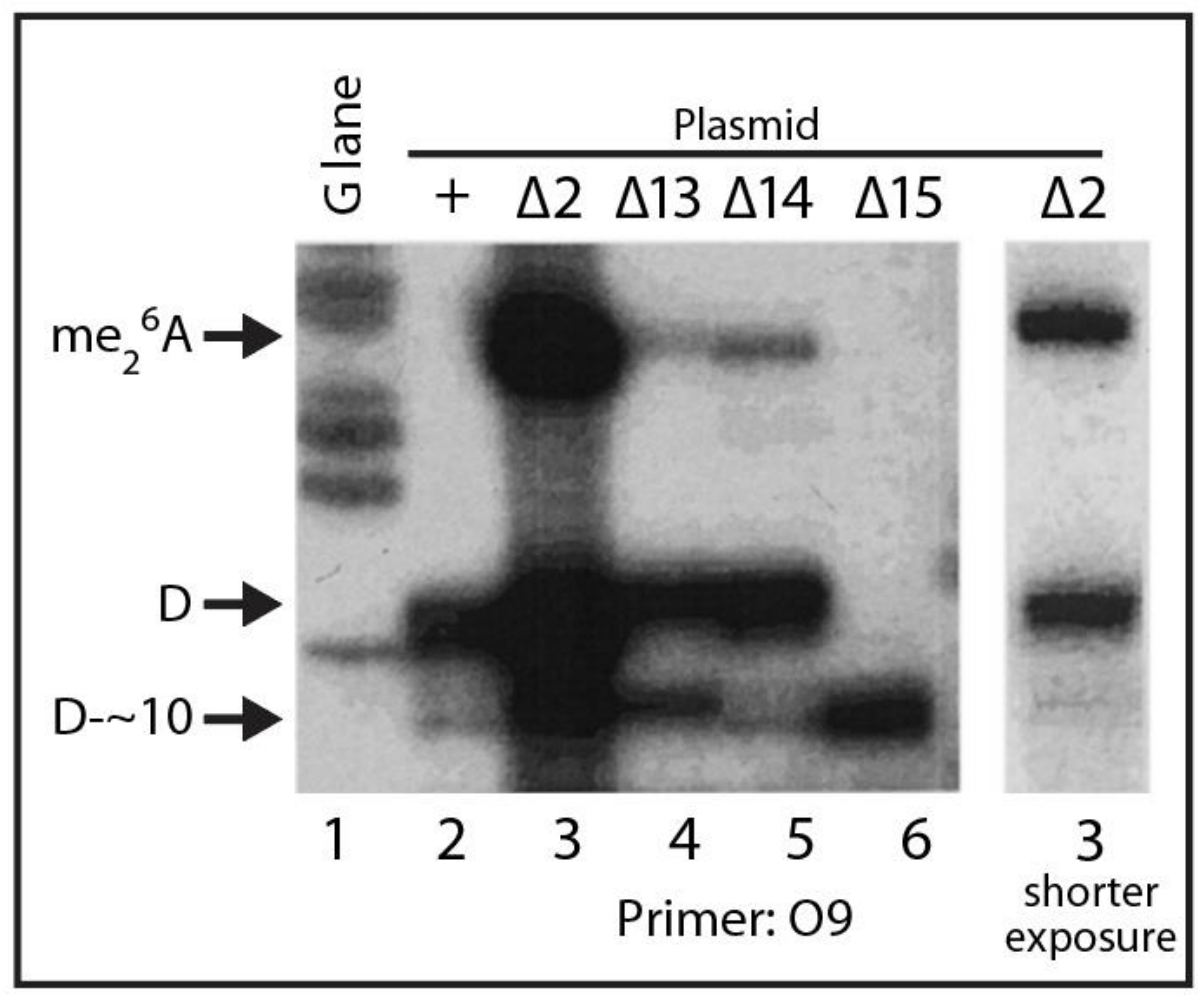


Figure 5. Mapping of 5' ends at the D-site made by processing of pre-rRNA from wildtype and the indicated deletion mutants. Host $\Delta$ rna12 carrying the indicated derivatives of pDK16-tag were grown at $25^{\circ}$ and shifted to $37^{\circ}$ for 6 hours. Total RNA was used for extension of primer O9 (Figure 1A). A G-lane made by dideoxy sequencing using total pDK16 DNA and primer O9 is shown as markers. The blot in the right shows lane 3 from a shorter exposure of the blot in lanes 1-6.

There is an additional interesting result from the primer extension experiment: RNA from $\Delta 2$ (and, to a lesser extent, $\Delta 13$ and $\Delta 14$ ) generates a longer band whose length corresponds to the position of two adenosines near the end of 18S rRNA that are dimethylated in the mature rRNA [33] (Figure 5, lanes 3-5). This band is not visible with the wildtype RNA, presumably because the D-cleavage normally occurs at a faster rate, thereby preceding the A-methylation. We conclude that in the $\Delta 2, \Delta 13$ and $\Delta 14$ mutants, the $D$ site cleavage is delayed relative to A-dimethylation, and that 3 ' end maturation of $18 \mathrm{~S}$ rRNA is not a prerequisite for dimethylation. Similar results were seen previously in A2 site mutants [18, 30].

Deletion of the gene for the 5'>3' exoribonuclease, $X R N 1$, has been shown to stabilize ITS1 processing fragments [34]. To gain further insight into the processing of the $\Delta 2$ pre-rRNA, we analyzed the accumulation of ITS1 rRNA fragments in a $\Delta x r n 1$ derivative of $\Delta r n a 12$ harboring pDK16-tag or pDK16-tag $\Delta 2$. RNA was prepared at 4 and 6 hours after a shift to the non-permissive temperature and analyzed on northern blots of agarose and acrylamide gels probed with 0453 (Figure 6A), which is complementary to the region of ITS1 just downstream of the D cleavage site (Figure 1A and Table 1). As previously observed [34], the elimination of Xrn1 activity with the wildtype ITS1 plasmid results in the accumulation of an RNA generated from cleavage at sites $D$ and A2 (D-A2) (Figure 6A, lanes 1-2, and lanes 7-8). At 4 hours post-temperature shift, the $\Delta 2$ mutant also contained accumulated D-A2 RNA; we attribute that to residual but decreased transcription of the chromosomal rRNA genes by Pol I as it was undergoing inactivation after the temperature shift. However, the blot also revealed a longer fragment, labelled $X$, derived from the processing of ITS1 from mutant $\Delta 2$ (Figure $6 \mathrm{~A}$, lanes 3-4 and lanes 9-10). By 6 hours, the D-A2 fragment was virtually gone, and only band $X$ was visible (Figure $6 A$, lanes $5-6$ and lanes 11-12). Furthermore, the wildtype 
$20 \mathrm{~S}$ (pre-18S) band generated by cleavage at A2 (Figure 1; barely visible in Figure 6A, lanes 1-2) was replaced in the $\Delta 2$ mutant with a new "24S" slower moving band (Figure $6 \mathrm{~A}$, lanes 3-6. This RNA may be the same fragment we have observed in an RNase MRP mutant, which extends from the 5' end of $18 \mathrm{~S}$ to the 3 ' end (E site) of 5.8S rRNA $[20,27]$. Finally, several bands between $X$ and 20 S were observed after the temperature shift of $\Delta r n a 12 \Delta x r n 1 / p D K \Delta 2$, indicating ectopic cleavage of $18 \mathrm{~S} r R N A$ (Figure 6A, compare lanes 3-6 with lanes 1-2). 


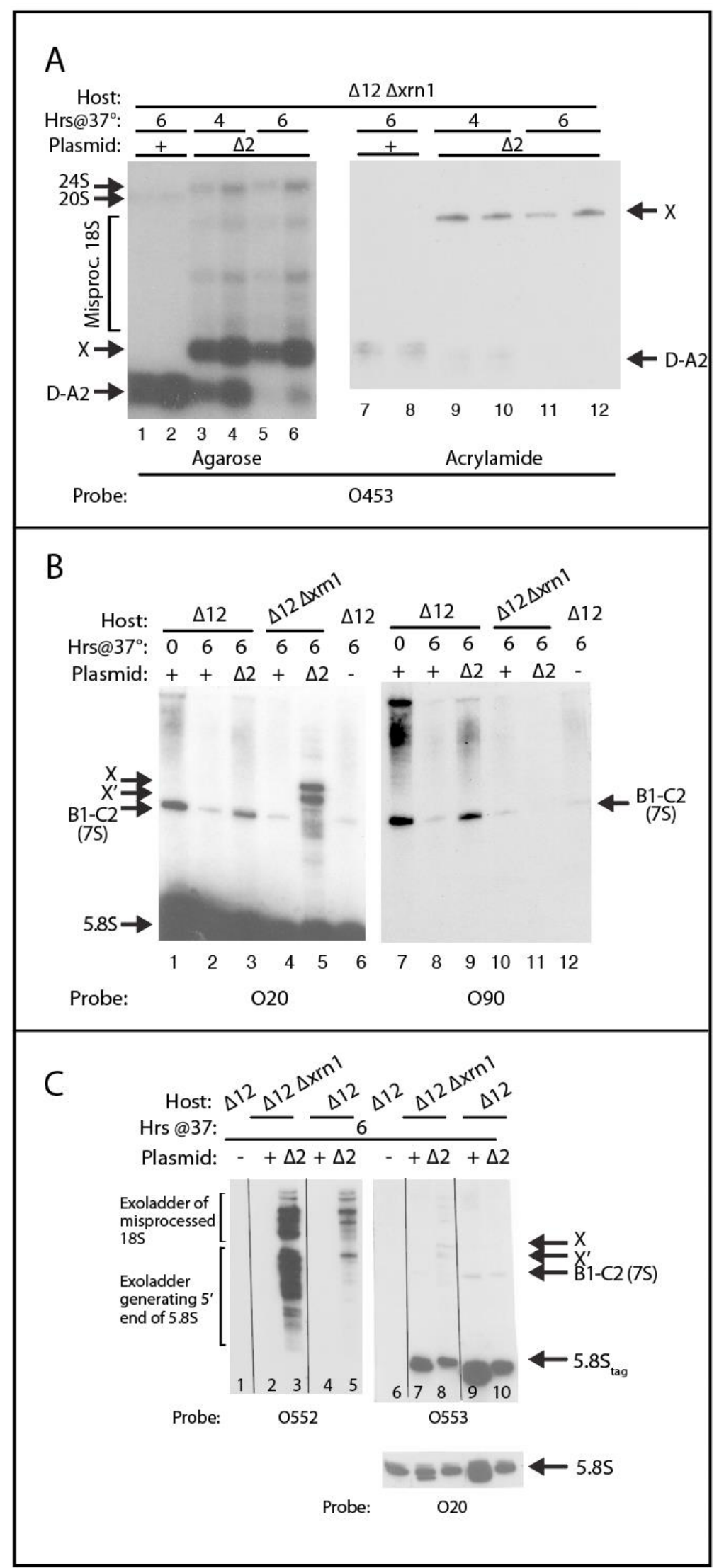


Figure 6. Characterization of intermediates in the processing of $\triangle 2$ pre-rRNA. Total RNA from $\Delta r n a 12 X R N 1$ or $\Delta r n a 12 \Delta x r n 1$ carrying pDK16-tag with wildtype or $\Delta 2$ rRNA genes was extracted from cells grown at $25^{\circ}$ (time 0 ) or after cells were shifted to $37^{\circ}$ for 4 or 6 hours. The RNA was fractionated by agarose or acrylamide gel electrophoresis and analyzed by northern blots. (A) The wildtype D-A2 wildtype intermediate is replaced with a longer intermediate $(X)$ in $\Delta 2$ processing. Agarose (left) and acrylamide gels (right) were blotted and probed with 0453 . (B) The $X$ and $X$ ' intermediates in processing of $\Delta 2$ pre-rRNA include the 3' end of 5.8S rRNA. Acrylamide gels were blotted and probed with $\mathrm{O} 20$ or O90. (C) The $\Delta 2$ pre-rRNA processing intermediates are subject to exonuclease maturation. An acrylamide gel was blotted and probed with 0552 (lanes 1-5), O553 (lanes 6-10), or O20 (5.8S slice of the blot showing the $5.8 \mathrm{~S}$ bands in lanes 6-10). All images in panel $\mathbf{C}$ came from the same blot, probed sequentially and stripped in between using each probe; see text for details.

Given the size of the $\Delta 2$ deletion, that $\Delta 2$ pre-rRNA is cleaved at the proper D site, and the fact that band $X$ is longer than $D-A 2$, the $X$ fragment must extend downstream of ITS1. We confirmed this by probing a blot of an acrylamide gel with the 5.8S-specific probe 020 . The blot of RNA from $\Delta r n a 12 \Delta x r n 1 / p D K 16-\Delta 2$-tag revealed two fragments, one of which corresponds to $X$. The other, called X', migrates slightly faster than $X$, but was not seen in the northern probed by 0453 ; it therefore must have a 5 ' end downstream of the $D$ site (Figure 6B, lane 5). Neither of the $X$ and $X$ ' bands hybridize to the 090 probe, complementary to the upstream part of ITS2 (Figure 6B, lane 11), suggesting that both $X$ and $X$ ' have 3 ' ends at or close to the 3 ' end of $5.8 S$ rRNA (site E). Probing of the blot with $\mathrm{O} 90$ or $\mathrm{O} 20$ also reveals a band in the $\Delta r n a 12$ $X R N 1 / p D K 16$-tag or the $\Delta r n a 12 X R N 1 / p D K 16$-tag $\Delta 2$ RNA corresponding to the $7 S$ prerRNA (Figure 1A), which is the 3' extended precursor for 5.8S rRNA bracketed by the $\mathrm{B} 1$ and $\mathrm{C} 2$ sites (Figure 6B lanes 1-3 and 7-9). This band is also seen in $\Delta r n a 12$ $\Delta x r n 1 / p D K 16-$ tag (Figure 6B, lanes 4 and 10). However, since the 75 band is not seen in $\Delta r n a 12 \Delta x r n 1 / p D K 16$-tag $\Delta 2$ probed with 090 , we conclude that, in the absence of Xrn1 activity, the 3 ' ends of $X$ and $X$ ' must be formed before the 5' end of $5.8 S$ is processed (Figure 6B, lane 11).

The probing of RNA from $\Delta r n a 12 \Delta x r n 1 / p D K 16$-tag $\Delta 2$ with $\mathrm{O} 20$ also revealed a series of bands below X and X' (Figure 6B, lane 5), suggesting that the two $X$ and X' RNA fragments are gradually shortened by exonuclease processing. To investigate this further, we designed a probe (0552) specific to RNA intermediates that include the $\Delta 2$ 
deletion (Figure 1A). This oligonucleotide is complementary to 9 nucleotides upstream and 11 nucleotides downstream of the $\Delta 2$ deletion in ITS1. Transcripts that encompass the $\Delta 2$ deletion can form a stable 20-basepair uninterrupted helix with the probe, while transcripts that do not contain the deletion are only able to form a $9 \mathrm{bp}$ or $11 \mathrm{bp}$ uninterrupted helix with 0552 , or a hybrid interrupted by a large loop, neither of which is stable enough to generate a hybrid under our hybridization conditions. As seen in Figure 6C, lanes 3 and 5, the 0552 oligonucleotide only hybridizes to RNA from strains harboring pDK16-tag $\Delta 2$, confirming that 0552 specifically reveals transcripts that span the $\Delta 2$ deletion. Moreover, RNA from both the $\Delta x r n 1$ and $X R N 1$ strains containing pDK16 $\Delta 2$ form exonuclease degradation "ladders" with 0552 (Figure $6 \mathrm{C}$, lanes 3 and 5, respectively), but the ladders in RNA from the $x r n 1 \Delta$ are much stronger (compare lane 3 with lane 5). Probing of the same blot with 0553 , complementary to tagged 5.8S rRNA, shows that there were similar amounts of tagged 5.8S rRNA in both samples (Figure $6 C$, lanes 8 and 10). Therefore, we ascribe the increased intensity in lane 3 relative to lane 5 to a slower degradation rate of ITS1 fragments when the Xrn1 endonuclease is absent, leading to a greater accumulation of the intermediates [35]. We conclude that Xrn1 is the primary exonuclease generating the ladders and that, in the absence of the Xrn1 nuclease, the degradation is performed by another, slower 5'>3' exonuclease.

Comparison of lanes 3 and 5 with lane 8 in Figure $6 \mathrm{C}$ shows that the bands at the top of the lower cluster of exonuclease products have an electrophoretic mobility similar to the $X$ and $X$ ' bands, while the lower bands correspond more or less to the mobility of $5.8 \mathrm{~S} \mathrm{~L}$. These results suggest that the lower ladders represent "trimming" of $X$ and X' RNA to form the mature 5' end of $5.8 \mathrm{~S}_{\mathrm{L}}$ (Figure $6 \mathrm{C}$, lanes 3 and 5 ). The upper ladder may represent trimming of RNA formed by misprocessing of $18 \mathrm{~S}$ rRNA.

\subsection{8S rRNA with 5' extended ends are incorporated into functional ribosomes} Since $\Delta 2$ rRNA supports growth at the non-permissive temperature of strain $\Delta r n a 12$, when the pDK16-tag $\Delta 2$ plasmid is the only source of rRNA, functional ribosomes must be formed from the pre-rRNA containing the $\Delta 2$ deletion. We were curious to see if any of the 5' extended 5.8S rRNA in X, X', and the exonuclease ladders are removed before or after the 5.8S rRNA is incorporated into ribosomes. Therefore, we fractionated by 
sucrose gradients whole-cell extracts of $\Delta r n a 12 \Delta x r n 1 / p D K 16$-tag $\Delta 2$ (Figure 7). RNA extracted from each sucrose gradient fraction was analyzed on a northern blot probed with 0576 , which visualizes the tagged 5.8S rRNA (Figure 1B). The results demonstrate that the $5.8 \mathrm{~S} \mathrm{~L}$ processed from the $\triangle 2$ pre-rRNA is incorporated into $60 \mathrm{~S}$ and $80 \mathrm{~S}$ ribosomes as well as polysomes (Figure 7, lanes 14-19 and lanes 20-27, respectively). Additionally, probing with 0552 showed that the 5'-extended 5.8S RNA transcripts ("exoladders") are also incorporated into ribosomes, including polysomes (Figure 7, lanes 42-55). In other words, the extensions do not preclude incorporation of the 5.8Scontaining rRNA into functional ribosomes. The distribution of transcripts of different lengths in the exonuclease ladders is somewhat different in the transcripts extracted from ribosomes compared to the total RNA extracted in the presence of hot phenol (Figure 7, lanes 29 and 56, see also Figure 6C, lanes 3 and 5), presumably because hot phenol used for RNA extraction from whole cells inactivates enzymatic activity immediately upon cell lysis, while some enzymatic activities, including exonucleases, may continue to operate in the crude lysates despite keeping the lysates on ice. Interestingly, "24S" rRNA containing the $\Delta 2$ mutant ITS1 is seen in complexes that sediment relatively slowly (Figure 7, fractions 2-8), but not in mature ribosomes. Thus, it appears that the "24S" transcript may be incorporated into slowly-sedimenting assembly intermediates (i.e., assembly intermediates in the early part of the assembly pathway) and that the 18S part is separated from the ITS1-5.8S part before ribosomes become functional. 


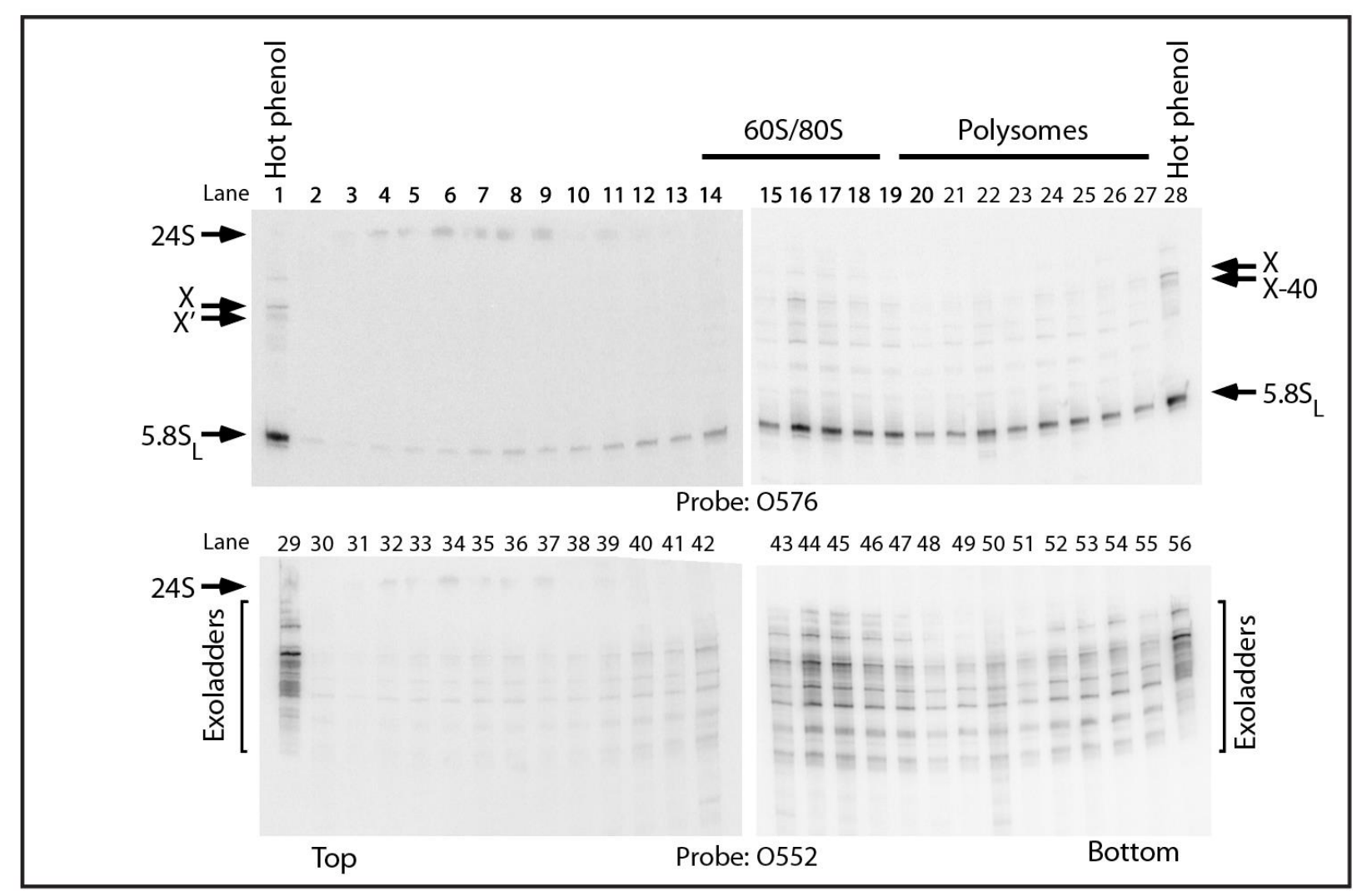

Figure 7. 5' extended 5.8S rRNA is incorporated into $60 \mathrm{~S}, 80 \mathrm{~S}$ and polysomal ribosomes. $\Delta r n a 12 \Delta x r n 1$ carrying pDK16-tag $\Delta 2$ rRNA genes was grown at $25^{\circ}$ and shifted to $37^{\circ}$ for 6 hours. Whole cell extracts were fractionated on sucrose gradients. RNA was then isolated from each fraction and subjected to acrylamide gel electrophoresis. Finally, RNA was transferred to nylon blots and probed with 0576 or 0552.

\section{Discussion}

3.1 Steps in the Xrn1-dependent path to the 5' end of $5.8 S_{L}$

The canonical scheme for pre-rRNA processing includes pathways to generate two different 5' ends, 7 nucleotides apart, of the 5.8S rRNA (Figure 1A). The 5' end of $5.8 \mathrm{Ss}$ (short $5.8 \mathrm{~S}$ ) is ostensibly created by cleavage at the A3 site in ITS1 by the endonuclease RNase MRP, followed by trimming by the exonucleases Rat1 and Rrp17 (Figures $1 \mathrm{~A}$ and 2) [16-21]. The 5 ' end of $5.8 \mathrm{~S} \mathrm{~L}$ (long $5.8 \mathrm{~S}$ ) is believed to be formed by an unknown endonuclease [22] (Figure 1A). However, a third pathway for 5.8S processing was implied by our previous observation that formation of $5.8 \mathrm{~S} \mathrm{~L}$ requires exonuclease Xrn1 at non-permissive temperature in a temperature-sensitive RNase 
MRP mutant ("Mini2") with a higher penetrance for RNase MRP inactivation than the rrp2-2- mutant used in Figure 4 [24].

In this report, we describe experiments that support the proposed Xrn1dependent path to 5.8SL. By deleting most of the downstream-half of ITS1, including the $\mathrm{A} 2$ and $\mathrm{A} 3$ sites ( $\Delta 2$ mutant, Figure $1 \mathrm{~B}$ ), we blocked the two canonical processing pathways. This, in turn, uncovered a third pathway, initiated by cleavage at the border between 18S and ITS1 ( $D$ site) followed by Xrn1 exonuclease trimming from $D$ to the B1L5' end (Figures 5, 6 and 8A). The removal of ITS1 sequences by Xrn1 occurs, at least in part, after the 5' end-extended 5.8S rRNA is incorporated into functional $60 \mathrm{~S}$ subunits, as indicated by the presence of ITS1 sequences from $\triangle 2$ pre-rRNA in the 60S$80 S$-polysome region of sucrose gradients (Figure 7). This observation is consistent with previous studies showing that large ribosomal subunits in both bacteria and yeast can be functional even if the rRNA is incompletely processed [36, 37].

\subsection{Switching between the pathways to the 5' end of 5.8S rRNA}

The processing of wildtype pre-rRNA at non-permissive temperature in the Mini2 RNase MRP temperature-sensitive mutant [24] shares two characteristics of the $5.8 \mathrm{~S} L$ formation from $\triangle 2$ pre-RNA in a wildtype RNase MRP background (this report). First, in both cases the ITS1 is not cleaved at either A2 or A3, and second, both require Xrn1 [see Figures 5 and 7 in [24]]. These shared features suggest that the processing of prerRNA in the absence of RNase MRP activity follows the pathway for $\triangle 2$ pre-rRNA processing. Furthermore, an exonuclease ladder is visible above $5.8 \mathrm{~S}\llcorner\mathrm{rRNA}$ after acrylamide gel electrophoresis of RNA prepared from cells grown at permissive temperature, i.e. while RNase MRP is active, suggesting that Xrn1 trimming is a normal pathway to $5.8 S_{\llcorner}$[see Figure 7 in [24]]. We conclude that the 5 ' end of 5.8S $\mathrm{L}$ can be formed either by Xrn1 or by an endonuclease while RNase MRP is active, but only the Xrn1-dependent pathway functions when RNase MRP is inactive (Figure 8B-C). The switch from accumulation of both forms of 5.8S rRNA to accumulation of only long $5.8 \mathrm{~S}$ rRNA can thus be triggered by inactivating RNase MRP, because that inactivation blocks $\mathrm{A} 2$ and $\mathrm{A} 3$ cleavage (Figure $8 \mathrm{C}$ ). Obviously, this impedes the canonical path to $5.8 \mathrm{~S}$ s, but it is not clear why it prevents the endonuclease cleavage at B1 L. An 
uncharacterized 5' end ("A4") slightly downstream of A2 was implicated in the endonuclease pathway [22], so perhaps this end cannot be generated without $A 3$ cleavage.

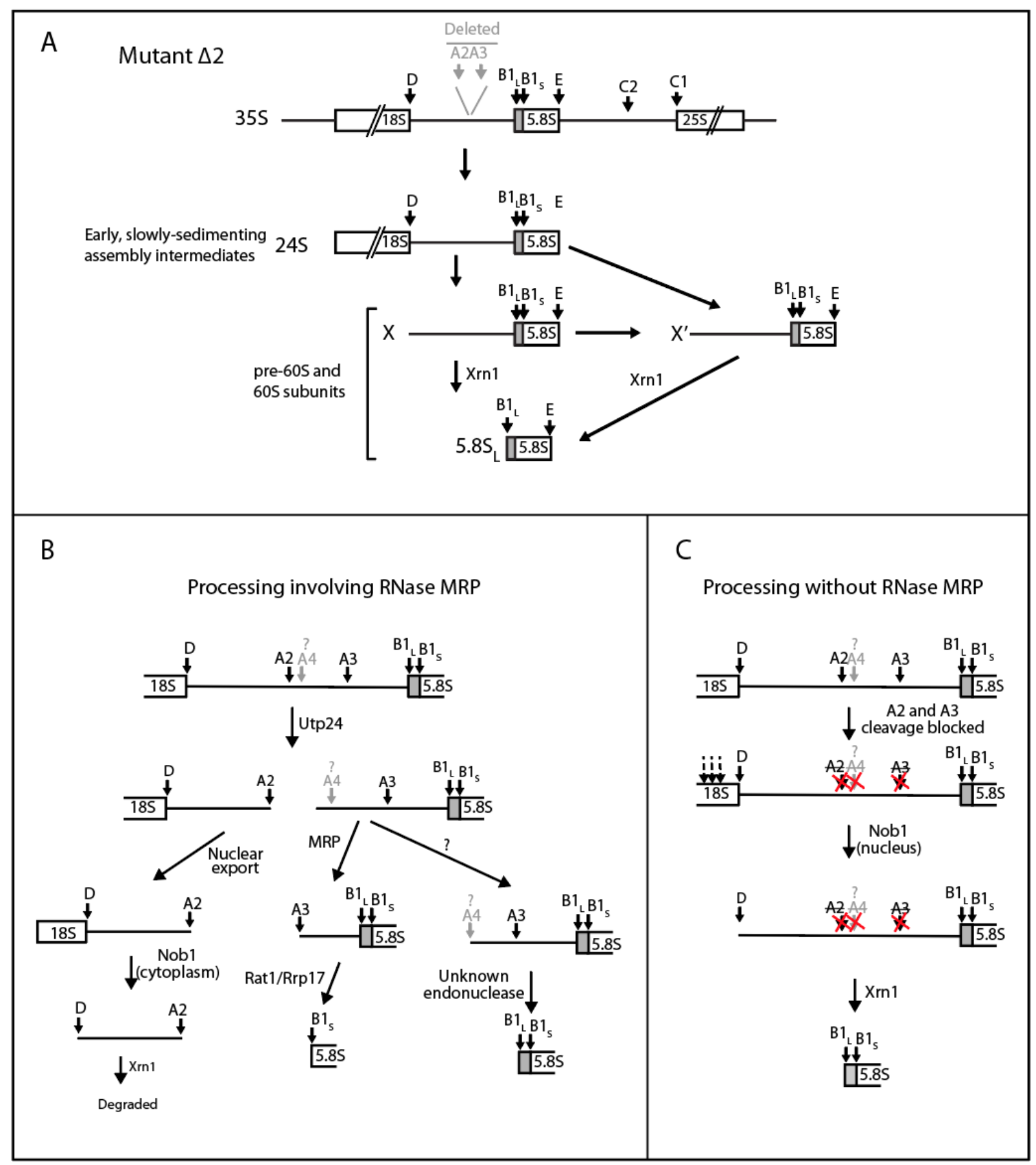


Figure 8. Models for ITS1 processing and switch between processing modes. (A) Xrn1dependent processing of $\Delta 2$ pre-rRNA. See text for details. (B) Processing involving RNase MRP (canonical model) Note, the path to $5.8 S_{\mathrm{L}}$ involves an intermediate with a 5 ' end (A4) that has not been characterized [22]. (C) Processing without RNase MRP participation. Inactivation of RNase MRP blocks cleavage at both A2 and A3, allowing Xrn1 exonuclease to degrade the entire region from $D$ to $B 1 \mathrm{~L}$. We propose that the processing of pre-rRNA occurs via $a$ competition of the models in panels $B$ and $C$ with the canonical model in panel $B$ being dominant during growth under laboratory conditions. If RNase MRP activity is decreased due to change of growth conditions or mutations, the kinetic mix of the models in panels $B$ and $C$ switches in the direction of model $\mathrm{C}$. In the extreme case of total inactivation of RNase MRP, processing occurs exclusively via the model in $\mathrm{C}$.

The canonical role of RNase MRP in 5.8Ss processing (Figure 1) is based on two observations. First, RNase MRP cleaves ITS1 rRNA at the A3 site in vitro [38-40], and second, accumulation of $5.8 \mathrm{~S}$ s is decreased in RNase MRP mutants, ostensibly because of a decreased rate of RNase MRP cleavage at A3. If that were the case, no 5.8Ss should be made from pre-rRNA lacking the A3 site. However, processing of 5.8Ss is accomplished from $\Delta 14, \Delta 15$, and $\Delta 16$ pre-rRNAs in normal amounts in an RNase MRP wildtype strain (RRP2), but in reduced amounts in the rrp2-2 RNase MRP mutant (Figure 4), even though these pre-rRNAs should not be subject to RNase MRP cleavage due to the deletion of the A3 site (Figure 4). This suggests that the canonical explanation for the role of RNase MRP in the switch between production of $5.8 \mathrm{~S}$ s and $5.8 S_{L}$ cannot be correct. Rather, we suggest that RNase MRP mediates the switch between $5.8 S_{s}$ and $5.8 \mathrm{~S} \mathrm{~L}$ by an indirect, rather than a direct, mechanism.

It should be noted, however, that even though 8 nucleotides around the A3 consensus sequence contact the RNase MRP binding pocket, only two nucleotides in the consensus sequence have a strong effect on the rate of cleavage [38, 39, 41]. Thus, we cannot exclude ectopic RNase MRP cleavage of $\Delta 14, \Delta 15$, and $\Delta 16$ in the $R R P 2$ strain, although if this were the case, we would have expected that such ectopic cleavage should also suppress the effect of the inhibition of A3 cleavage in the $\Delta 18$ mutant.

Interestingly, processing of $\Delta 17$ pre-rRNA also generates little 5.8Ss rRNA, even though this deletion leaves the A3 site intact. Conceivably, the Rat1 and Rrp17 exonucleases fail to stop at what would be the mature 5 ' end of $5.8 \mathrm{~S}$ s when the 
proposed hairpin formed by the distal region of ITS1 (ITS1 nucleotide 330 through position 27 of $5.8 \mathrm{~S}$ s; see Figure 2 ) and proximal $5.8 \mathrm{~S}$ sequences ( $5.8 \mathrm{~S}$ s positions $1-24$ ) is disrupted, even though the remaining part of the hairpin is sufficient to stop exonuclease degradation at the 5 ' end of $5.8 \mathrm{~S}$. Overall, our analysis of $5.8 \mathrm{~S}$ s formation from the ITS1 deletion pre-rRNA in RNase MRP wildtype and mutant strains raises doubts about the canonical pathway to the 5' end of 5.8Ss.

\subsection{Why is RNase MRP essential?}

The RNA component of RNase MRP is essential for rRNA synthesis and growth in both yeast and humans [23, 24]. Indeed, mutations in this RNA have been implicated in human diseases [42, 43]. However, the essential nature of RNase MRP RNA cannot be rationalized based on our understanding of its role in rRNA synthesis, since both $5.8 \mathrm{~S} s$ and $5.8 \mathrm{~S} \mathrm{~L}$ get incorporated into functional ribosomes as indicated by the facts that the $\Delta 2$ mutant is viable with essentially no $5.8 \mathrm{~S}$ s (Figures1-4) and subunits containing $5.8 S_{\llcorner}$engage in polysome formation (Figure 7 ). Furthermore, even though early cleavages in the pre-rRNA can be detected by northern analysis at non-permissive temperature of the temperature sensitive "Mini2" mutant of RNase MRP, the processing generates neither 18S nor 25S rRNA mature rRNA [24]. Yet, 18S and 25S are evidently accumulating while the canonical ITS1 processing is bypassed, since the $\Delta 2$ mutant is viable (Figure 7). Finally, it is not clear why A2 cleavage fails in the absence of RNase MRP activity [24], since A2 is cleaved by Utp24, which is a component of the 90S pre40S particle [12]. Moreover, base substitutions in the A2 and A3 cleavage sites indicate that $A 2$ cleavage is not dependent on A3 cleavage [18].

Taken together, these observations lead us to conclude that RNase MRP has other, as yet unidentified but essential, role(s) related to pre-rRNA processing. The notion, suggested above, that RNase MRP might control processing of one or more snoRNAs and mRNAs for ribosome processing factors may explain the essential nature of RNase MRP. In fact, RNase MRP has already been implicated in processing of other classes of mRNAs [44-47].

\subsection{The 3' end maturation of $18 \mathrm{~S}$ rRNA}


The 3' end of 18S rRNA is matured by Nob1 endonuclease cleavage at the D-site of the $20 S$ pre-rRNA [48]. Nob1 binds, together with partner proteins Pno1 and Nop9, to both $18 \mathrm{~S}$ and ITS1 sequences of the 20S pre-rRNA in the nuclear pre-40S particle; cleavage is then prevented until the partner proteins are removed by helicases [49-51]. The $\Delta 4$ and $\Delta 7$ deletions remove parts of the pre-rRNA binding sites for Non1 and partner proteins, which may account for the negative effect on growth of those mutations.

Because the D-A2 fragment can be found in the cytoplasm [52, 53], D cleavage is assumed to occur after export of $40 \mathrm{~S}$ ribosomal precursors particles from the nucleus to the cytoplasm. However, this observation does not exclude that $D$ cleavage could also occur in the nucleus prior to export of the pre-40S, since Nob1 binds to pre-40S particles in the nucleus and its inhibitory proteins may conceivably be removed by nuclear helicases. Indeed, our results suggest that D cleavage does occur in the nucleus. Since both known endonuclease targets within ITS1 (A2 and A3) have been deleted in $\Delta 2$, the recognized mechanism for separating the rRNA moieties destined for $40 \mathrm{~S}$ and $60 \mathrm{~S}$ ribosomes is blocked. Therefore, we propose that, in the $\Delta 2$ mutant, the rRNA for the two subunits is instead separated by D cleavage. However, since nuclear export of the large and small ribosomal subunits requires different export factor [54], it seems unlikely that $18 \mathrm{~S}$ and $5.8 \mathrm{~S} / 25 \mathrm{~S}$ would be exported together in a single preribosome particle. Thus, it is reasonable to suppose that $\mathrm{D}$-cleavage is a nuclear function during processing of $\Delta 2$ pre-rRNA. The cellular location of $D$ cleavage may be determined by kinetic competition between nuclear export of pre-40S and cleavage at D. If the export is fast, Nob1 cleavage will occur predominantly in the cytoplasm, but during slow pre-40S export, Nob1 cleavage may become a nuclear function. In the case of $\triangle 2$ pre-rRNA processing, export of pre-40S is likely blocked until the precursors for $40 S$ and 605 have been separated.

Previous base substitution experiments suggested that base-pairing of ITS1 sequences with nucleotides in the distal part of 18S rRNA block access for the Nob1 nuclease to the D site until A2 has been cleaved [55]. The ITS1 sequences participating in the proposed ITS1-18S base-pairing are deleted in the $\Delta 2, \Delta 13$, and $\Delta 14$ mutants; consequently, the proposed linkage of D-cleavage to A2-cleavage cannot function in these mutants. Thus, the proposed structure switch linking D-cleavage to A2 cleavage 
can be bypassed. However, the ectopic cleavages within $18 \mathrm{~S}$ seen in these mutants may normally be prevented during processing of wildtype pre-RNA by the proposed ITS1-18S base-pairing. Interestingly, the D-cleavage in the $\Delta 15$ mutant occurs $\sim 10$ nucleotides 3' to the correct D-site, even though the deletion is located 250-299 nucleotides downstream of $D$. Perhaps the deletion removes a binding site for a ribosomal assembly factor, or indirectly causes a refolding of the ITS1 sequences closer to the D-site.

\subsection{Diversity of rRNA processing pathways}

Like the pathway for bypass of ITS1 A2 and A3 cleavage described here, other steps for ribosome formation can also be bypassed by suppressor mutations. For example, release of Nsa1, a participant in restructuring one of the nucleolar pre-60S particles, requires the Rix7 ATP-helicase to be released from the pre-60S particle, but this step can be circumvented by mutations in EBP2 and MAK5 that eliminate the need for Nsa1 in facilitating pre-60S restructuring [56]. Similarly, a mutational change in the multifunctional protein Rrp5 can bypass A2 cleavage [57]. Moreover, mutations in Rsr1, Rpf2, or uL5, proteins that form a ribosomal subparticle with the 5S rRNA before docking of 5S rRNA-uL5 in the pre-60S particle, can suppress the need for Cgr1 in the final positioning of $5 S$ rRNA in the nascent pre-60S [58]. The theme for the bypass pathways may be that they co-exist in wildtype strains, but are kinetically noncompetitive. The suppressor mutations may change the kinetics of the bypass reaction relative to the canonical pathways, enabling these alternative pathways to become kinetically significant.

\section{Materials and Methods}

\subsection{Strains and growth conditions}

Yeast strains used are shown in Table 2. In $\Delta$ rna135, the gene for the largest RNA polymerase subunit is disrupted, which inactivates RNA Pol I at all temperatures [28]. The pNOY 102 plasmid in $\Delta$ rna135 was replaced by pDK16 or pDK $16 \Delta 2$ by transformation and selection for TRP+ followed by counter-selection of pNOY102 
(URA3) by growth on 5 -fluoroorotic acid. Cultures of $\Delta$ rna 135 were grown at $30^{\circ}$. In $\Delta$ rna12, disruption of the gene for the smallest subunit of RNA-Pol I bestows temperature sensitivity for growth [29]. An XRN1 deletion derivative of the temperaturesensitive strain $\Delta r n a 12$ (called $\Delta r n a 12 \Delta x r n 1$ ) was constructed by transforming $\Delta r n a 12$ with a PCR fragment made from chromosomal DNA of an xrn1 $\triangle \mathrm{Bgl1}:: U R A 3$ strain [59] and selecting for uracil prototrophy. Cultures of $\Delta r n a 12$ or its $\Delta x r n 1$ derivative carrying the indicated plasmid were grown at $25^{\circ}$ in supplemented synthetic medium [60] lacking tryptophan and including $\mathrm{Cu}^{2+}\left(0.1 \mathrm{mM} \mathrm{CuSO}_{4}\right.$, the inducer of the CUP1 promoter), and shifted to $37^{\circ}$ for 4 or 6 hours as indicated.

Table 2. Strains and plasmids

\begin{tabular}{|l|l|l|}
\hline Name & Genotype & References \\
\hline $\begin{array}{l}\Delta \text { rna12 alias } \\
\text { NOY504 }\end{array}$ & $\begin{array}{l}\text { MATa rm4 (rna12)::LEU2 ade2-101 ura3-1 } \\
\text { trp1-1 leu2-3,112 his3-101 can1-100 }\end{array}$ & {$[29]$} \\
\hline $\begin{array}{l}\Delta \text { rna135 alias } \\
\text { NOY408-la }\end{array}$ & $\begin{array}{l}\text { MATa rpa135::LEU2 ade2-1 ura3-1 his3-10 } \\
\text { 1trp1-1 leu2-112 can1-100/pNOY102 }\end{array}$ & {$[28]$} \\
\hline$\Delta$ rna135/pDK16 & $\begin{array}{l}\text { MATa rpa135::LEU2 ade2-I ura3-I his3-II trpl-1 } \\
\text { leu2-3,112 can1-100/pDK16 }\end{array}$ & This study \\
\hline YLL53 & MATa ade2-101 his3 200 ura3-52 tyr1 RRP2 & {$[20]$} \\
\hline YLL54 & $\begin{array}{l}\text { MATa, ade2-101, his3 200, ura3-52, lys2, rrp2- } \\
2\end{array}$ & {$[20]$} \\
\hline pDK16 & $\begin{array}{l}\text { YEplac112 carrying the rRNA transcription } \\
\text { unit expressed from the CUP1 promoter }\end{array}$ & {$[30]$} \\
\hline pDK16-tag & pDK16 with tagged 5,8S gene (see text) & This study \\
\hline
\end{tabular}

\subsection{Plasmids and oligonucleotides}

Plasmid pDK16 [30], a yeast-E.coli shuttle plasmid containing both 2-Micron and ColEI origins of replication, harbors a wild type copy of the yeast rRNA transcription unit controlled by the CUP1 promoter. pNOY102 [28] carries a wildtype rRNA transcription unit expressed from the GAL7 promoter. Deletions were made in pDK16 by joining together PCR fragments with anchors of restriction enzyme recognition sites made with pNOY102 as template. 
Oligonucleotides used for northern and primer extension analyses are listed in Table 1. Two probes (O553 and O576) were made for the Sp tag on pDK16. Both work for northern analysis, but $\mathrm{O} 553$ has a propensity for hairpin formation that appears to limit its capacity to work in primer extension.

\subsection{Other procedures}

Gel electrophoresis, blotting, and sucrose gradient procedures were performed as described [27, 61]. Total RNA was extracted from cells as described previously [27]. RNA was extracted from sucrose gradient fractions by phenol extraction followed by a phenol/chloroform/isoamyl alcohol extraction and concentrated by ethanol precipitation.

\section{Acknowledgments}

This work was supported by a grant from the National Institute for General Medical Science 54876. We thank Susan Fretz for superb technical assistance. 


\section{References}

1. Maaløe, O.; Kjeldgaard, N. O., Control of Macromolecular Synthesis. Benjamin: New York, 1966.

2. Kjeldgaard, N. O.; Maaloe, O.; Schaechter, M., The transition between different physiological states during balanced growth of Salmonella typhimurium. J Gen Microbiol 1958, 19, (3), 607-16.

3. Warner, J. R., The economics of ribosome biosynthesis in yeast. Trends Biochem Sci 1999, 24, (11), 437-40.

4. Cerezo, E.; Plisson-Chastang, C.; Henras, A. K.; Lebaron, S.; Gleizes, P. E.; O'Donohue, M. F.; Romeo, Y.; Henry, Y., Maturation of pre-40S particles in yeast and humans. Wiley interdisciplinary reviews. RNA 2019, 10, (1), e1516.

5. Tomecki, R.; Sikorski, P. J.; Zakrzewska-Placzek, M., Comparison of preribosomal RNA processing pathways in yeast, plant and human cells - focus on coordinated action of endo- and exoribonucleases. FEBS Lett 2017, 591, (13), 1801-1850.

6. Bohnsack, K. E.; Bohnsack, M. T., Uncovering the assembly pathway of human ribosomes and its emerging links to disease. EMBO J 2019, 38, (13), e100278.

7. Klinge, S.; Woolford, J. L., Jr., Ribosome assembly coming into focus. Nat Rev Mol Cell Biol 2019, 20, (2), 116-131.

8. Bassler, J.; Hurt, E., Eukaryotic Ribosome Assembly. Annu Rev Biochem 2019, 88, 8.1-8.26.

9. Woolford, J. L., Jr.; Baserga, S. J., Ribosome biogenesis in the yeast Saccharomyces cerevisiae. Genetics 2013, 195, (3), 643-81.

10. Axt, K.; French, S. L.; Beyer, A. L.; Tollervey, D., Kinetic analysis demonstrates a requirement for the Rat1 exonuclease in cotranscriptional pre-rRNA cleavage. PLoS One 2014, 9, (2), e85703.

11. Talkish, J.; Biedka, S.; Jakovljevic, J.; Zhang, J.; Tang, L.; Strahler, J. R.; Andrews, P. C.; Maddock, J. R.; Woolford, J. L., Jr., Disruption of ribosome assembly in yeast blocks cotranscriptional pre-rRNA processing and affects the global hierarchy of ribosome biogenesis. RNA 2016, 22, (6), 852-66.

12. An, W.; Du, Y.; Ye, K., Structural and functional analysis of Utp24, an endonuclease for processing $18 \mathrm{~S}$ ribosomal RNA. PLoS One 2018, 13, (4), e0195723.

13. Kufel, J.; Dichtl, B.; Tollervey, D., Yeast Rnt1p is required for cleavage of the preribosomal RNA in the 3' ETS but not the 5' ETS. Rna 1999, 5, (7), 909-17.

14. Bleichert, F.; Granneman, S.; Osheim, Y. N.; Beyer, A. L.; Baserga, S. J., The PINc domain protein Utp24, a putative nuclease, is required for the early cleavage steps in 18S rRNA maturation. Proc Natl Acad Sci U S A 2006, 103, (25), 9464-9.

15. Wells, G. R.; Weichmann, F.; Colvin, D.; Sloan, K. E.; Kudla, G.; Tollervey, D.; Watkins, N. J.; Schneider, C., The PIN domain endonuclease Utp24 cleaves preribosomal RNA at two coupled sites in yeast and humans. Nucleic Acids Res 2016, 44, (18), 9016.

16. Henry, Y.; Wood, H.; Morrissey, J. P.; Petfalski, E.; Kearsey, S.; Tollervey, D., The $5^{\prime}$ end of yeast $5.8 \mathrm{~S}$ rRNA is generated by exonucleases from an upstream cleavage site. EMBO J. 1994, 13, 2452-2463. 
17. Oeffinger, M.; Zenklusen, D.; Ferguson, A.; Wei, K. E.; El Hage, A.; Tollervey, D.; Chait, B. T.; Singer, R. H.; Rout, M. P., Rrp17p is a eukaryotic exonuclease required for 5 ' end processing of Pre-60S ribosomal RNA. Molecular cell 2009, 36, (5), 768-81.

18. Allmang, C.; Henry, Y.; Morrissey, J. P.; Wood, H.; Petfalski, E.; Tollervey, D., Processing of the yeast pre-rRNA at sites $A(2)$ and $A(3)$ is linked. Rna 1996, 2, (1), 63-73.

19. Schmitt, M. E.; Clayton, D. A., Nuclear RNase MRP is required for correct processing of pre-5.8S rRNA in Saccharomyces cerevisiae. Mol. Cell. Biol. 1993, 13, 7935-7941.

20. Chu, S.; Archer, R. H.; Zengel, J. M.; Lindahl, L., The RNA of RNase MRP is required for normal processing of ribosomal RNA. Proc. Natl. Acad. Sci. USA 1994, 91, 659-663.

21. Granneman, S.; Petfalski, E.; Tollervey, D., A cluster of ribosome synthesis factors regulate pre-rRNA folding and 5.8S rRNA maturation by the Rat1 exonuclease. The EMBO journal 2011.

22. Faber, A. W.; Vos, H. R.; Vos, J. C.; Raue, H. A., 5'-end formation of yeast 5.8SL rRNA is an endonucleolytic event. Biochem Biophys Res Commun 2006, 345, (2), 796-802.

23. Goldfarb, K. C.; Cech, T. R., Targeted CRISPR disruption reveals a role for RNase MRP RNA in human preribosomal RNA processing. Genes Dev 2017, 31, (1), 59-71.

24. Lindahl, L.; Bommankanti, A.; Li, X.; Hayden, L.; Jones, A.; Khan, M.; Oni, T.; Zengel, J. M., RNase MRP is required for entry of $35 \mathrm{~S}$ precursor rRNA into the canonical processing pathway. Rna 2009, 15, (7), 1407-16.

25. Chu, S.; Zengel, J. M.; Lindahl, L., A novel protein shared by RNase MRP and RNase P. RNA 1997, 3, 382-391.

26. Lygerou, Z.; Mitchell, P.; Petfalski, E.; Seraphin, B.; Tollervey, D., The POP1 gene encodes a protein component common to the RNase MRP and RNase P ribonucleoproteins. Genes Dev 1994, 8, (12), 1423-33.

27. Lindahl, L.; Archer, R. H.; Zengel, J. M., A new rRNA processing mutant of Saccharomyces cerevisiae. Nucleic Acids Res 1992, 20, (2), 295-301.

28. Nogi, Y.; Yano, R.; Nomura, M., Synthesis of large rRNAs by RNA polymerase II in mutants of Saccharomyces cerevisiae defective in RNA polymerase I. Proc. Natl. Acad. Sci. USA 1991, 88, 3962-3966.

29. Nogi, Y.; Yano, R.; Dodd, J.; Carles, C.; Nomura, M., Gene RRN4 in Saccharomyces cerevisiae encodes the A12.2 subunit of RNA polymerase I and is essential only at high temperatures. Mol. Cell. Biol. 1993, 13, 114-122.

30. Lindahl, L.; Archer, R. H.; Zengel, J. M., Alternate pathways for processing in the internal transcribed spacer 1 in pre-rRNA of Saccharomyces cerevisiae. Nucleic Acids Res. 1994, 22, 5399-5407.

31. van Nues, R. W.; Rientjes, J. M.; van der Sande, C. A.; Zerp, S. F.; Sluiter, C.; Venema, J.; Planta, R. J.; Raue, H. A., Separate structural elements within internal transcribed spacer 1 of Saccharomyces cerevisiae precursor ribosomal RNA direct the formation of $17 \mathrm{~S}$ and $26 \mathrm{~S}$ rRNA. Nucleic Acids Res 1994, 22, (6), 912-9. 
32. Li, X.; Zaman, S.; Langdon, Y.; Zengel, J. M.; Lindahl, L., Identification of a functional core in the RNA component of RNase MRP of budding yeasts. Nucleic Acids Res 2004, 32, (12), 3703-11.

33. Lafontaine, D.; Delcour, J.; Glasser, A. L.; Desgres, J.; Vandenhaute, J., The DIM1 gene responsible for the conserved m6(2)Am6(2)A dimethylation in the 3'terminal loop of $18 \mathrm{~S}$ rRNA is essential in yeast. J. Mol. Biol. 1994, 241, (3), 4927.

34. Stevens, A.; Hsu, C. L.; Isham, K. R.; Larimer, F. W., Fragments of the internal transcribed spacer 1 of pre-rRNA accumulate in Saccharomyces cerevisiae lacking 5'-3' exoribonuclease 1. J. Bacteriol. 1991, 173, 7024-7028.

35. Lindahl, L., Intermediates and time kinetics of the in vivo assembly of Escherichia coli ribosomes. J Mol Biol 1975, 92, (1), 15-37.

36. Lawrence, M. G.; Shamsuzzaman, M.; Kondopaka, M.; Pascual, C.; Zengel, J. M.; Lindahl, L., The extended loops of ribosomal proteins UL4 and UL22 of Escherichia coli contribute to ribosome assembly and protein translation. Nucleic Acids Res 2016, 44, (12), 5798-810.

37. Rodriguez-Galan, O.; Garcia-Gomez, J. J.; Kressler, D.; de la Cruz, J., Immature large ribosomal subunits containing the 7S pre-rRNA can engage in translation in Saccharomyces cerevisiae. RNA Biol 2015, 12, (8), 838-46.

38. Esakova, O.; Perederina, A.; Quan, C.; Berezin, I.; Krasilnikov, A. S., Substrate recognition by ribonucleoprotein ribonuclease MRP. RNA 2011, 17, (2), 356-64.

39. Lan, P.; Zhou, B.; Tan, M.; Li, S.; Cao, M.; Wu, J.; Lei, M., Structural insight into precursor ribosomal RNA processing by ribonuclease MRP. Science 2020, 369, (6504), 656-663.

40. Lygerou, Z.; Allmang, C.; Tollervey, D.; Seraphin, B., Accurate processing of a eukaryotic precursor ribosomal RNA by ribonuclease MRP in vitro. Science 1996, 272, 268-270.

41. Perederina, A.; Li, D.; Lee, H.; Bator, C.; Berezin, I.; Hafenstein, S. L.; Krasilnikov, A. S., Cryo-EM structure of catalytic ribonucleoprotein complex RNase MRP. Nat Commun 2020, 11, (1), 3474.

42. Mattijssen, S.; Welting, T. J.; Pruijn, G. J., RNase MRP and disease. Wiley interdisciplinary reviews. RNA 2010, 1, (1), 102-16.

43. Ridanpaa, M.; van Eenennaam, H.; Pelin, K.; Chadwick, R.; Johnson, C.; Yuan, B.; vanVenrooij, W.; Pruijn, G.; Salmela, R.; Rockas, S.; Makitie, O.; Kaitila, I.; de la Chapelle, A., Mutations in the RNA Component of RNase MRP Cause a Pleiotropic Human Disease, Cartilage-Hair Hypoplasia. Cell 2001, 104, (2), 195203.

44. Mattijssen, S.; Hinson, E. R.; Onnekink, C.; Hermanns, P.; Zabel, B.; Cresswell, P.; Pruijn, G. J., Viperin mRNA is a novel target for the human RNase MRP/RNase P endoribonuclease. Cellular and molecular life sciences : CMLS 2011, 68, (14), 2469-80.

45. Steinbusch, M. M. F.; Caron, M. M. J.; Surtel, D. A. M.; Friedrich, F.; Lausch, E.; Pruijn, G. J. M.; Verhesen, W.; Schroen, B. L. M.; van Rhijn, L. W.; Zabel, B.; Welting, T. J. M., Expression of RMRP RNA is regulated in chondrocyte hypertrophy and determines chondrogenic differentiation. Sci Rep 2017, 7, (1), 6440. 
46. Aulds, J.; Wierzbicki, S.; McNairn, A.; Schmitt, M. E., Global identification of new substrates for the yeast endoribonuclease, RNase mitochondrial RNA processing (MRP). J Biol Chem 2012, 287, (44), 37089-97.

47. Gill, T.; Cai, T.; Aulds, J.; Wierzbicki, S.; Schmitt, M. E., RNase MRP cleaves the CLB2 mRNA to promote cell cycle progression: novel method of mRNA degradation. Mol Cell Biol 2004, 24, (3), 945-53.

48. Fatica, A.; Oeffinger, M.; Dlakic, M.; Tollervey, D., Nob1p is required for cleavage of the 3' end of 18S rRNA. Mol Cell Biol 2003, 23, (5), 1798-807.

49. Ameismeier, M.; Zemp, I.; van den Heuvel, J.; Thoms, M.; Berninghausen, O.; Kutay, U.; Beckmann, R., Structural basis for the final steps of human 40S ribosome maturation. Nature 2020, 587, (7835), 683-687.

50. Turowski, T. W.; Lebaron, S.; Zhang, E.; Peil, L.; Dudnakova, T.; Petfalski, E.; Granneman, S.; Rappsilber, J.; Tollervey, D., Rio1 mediates ATP-dependent final maturation of $40 S$ ribosomal subunits. Nucleic Acids Res 2014, 42, (19), 12189 99.

51. Zhang, J.; McCann, K. L.; Qiu, C.; Gonzalez, L. E.; Baserga, S. J.; Hall, T. M., Nop9 is a PUF-like protein that prevents premature cleavage to correctly process pre-18S rRNA. Nat Commun 2016, 7, 13085.

52. Udem, S. A.; Warner, J. R., The cytoplasmic maturation of a ribosomal precursor ribonucleic acid in yeast. J. Biol. Chem. 1973, 248, 1412-1416.

53. Moy, T. I.; Silver, P. A., Nuclear export of the small ribosomal subunit requires the ran-GTPase cycle and certain nucleoporins [In Process Citation]. Genes Dev 1999, 13, (16), 2118-33.

54. Pena, C.; Hurt, E.; Panse, V. G., Eukaryotic ribosome assembly, transport and quality control. Nat Struct Mol Biol 2017, 24, (9), 689-699.

55. Lamanna, A. C.; Karbstein, K., An RNA conformational switch regulates pre-18S rRNA cleavage. J Mol Biol 2011, 405, (1), 3-17.

56. Pratte, D.; Singh, U.; Murat, G.; Kressler, D., Mak5 and Ebp2 act together on early pre-60S particles and their reduced functionality bypasses the requirement for the essential pre-60S factor Nsa1. PLoS One 2013, 8, (12), e82741.

57. Torchet, C.; Hermann-Le Denmat, S., Bypassing the rRNA processing endonucleolytic cleavage at site A2 in Saccharomyces cerevisiae. RNA 2000, 6, (11), 1498-508.

58. Thoms, M.; Mitterer, V.; Kater, L.; Falquet, L.; Beckmann, R.; Kressler, D.; Hurt, E., Suppressor mutations in Rpf2-Rrs1 or Rpl5 bypass the Cgr1 function for preribosomal 5S RNP-rotation. Nat Commun 2018, 9, (1), 4094.

59. Larimer, F. W.; Stevens, A., Disruption of the gene $X R N 1$, coding for a 5' -> 3' exoribonuclease, restricts yeast cell growth. Gene 1990, 95, 85-90.

60. Sherman, F.; Lawrence, C. W.; Fink, G. R., Methods in yeast genetics. Cold Spring Harbor Laboratory: Cold Spring Harbor, N.Y., 1979; p 98 p.

61. Gregory, B.; Rahman, N.; Bommakanti, A.; Shamsuzzaman, M.; Thapa, M.; Lescure, A.; Zengel, J. M.; Lindahl, L., The small and large ribosomal subunits depend on each other for stability and accumulation. Life Sci Alliance 2019, 2, (2). 